OPEN ACCESS

Edited by:

Marc Strous,

University of Calgary, Canada

Reviewed by:

Efstathios D. Giaouris,

University of the Aegean, Greece

Akos T. Kovacs,

Friedrich Schiller University Jena,

Germany

${ }^{*}$ Correspondence:

Scott A. Rice

School of Biological Sciences and Singapore Centre on Environmental Life Sciences Engineering, Nanyang

Technological University, 60 Nanyang Drive,

SBS-01N-27 Singapore, Singapore rscott@ntu.edu.sg

Specialty section:

This article was submitted to Microbial Physiology and Metabolism,

a section of the journal

Frontiers in Microbiology

Received: 18 February 2015 Accepted: 04 August 2015

Published: 20 August 2015

Citation:

Periasamy S, Nair HAS, Lee KWK,

Ong J, Goh JQJ, Kjelleberg S and Rice SA (2015) Pseudomonas aeruginosa PAO1 exopolysaccharides are important for mixed species biofilm community development and stress tolerance.

Front. Microbiol. 6:851. doi: 10.3389/fmicb.2015.00851

\section{Pseudomonas aeruginosa PAO1 exopolysaccharides are important for mixed species biofilm community development and stress tolerance}

\author{
Saravanan Periasamy ${ }^{1}$, Harikrishnan A. S. Nair ${ }^{1,2}$, Kai W. K. Lee ${ }^{1}$, Jolene Ong ${ }^{1,3}$, \\ Jie Q. J. Goh ${ }^{1,3}$, Staffan Kjelleberg ${ }^{1,3,4}$ and Scott A. Rice ${ }^{1,3,5 *}$ \\ 'Singapore Centre on Environmental Life Sciences Engineering, Nanyang Technological University, Singapore, Singapore, \\ ${ }^{2}$ Interdisciplinary Graduate School, Nanyang Technological University, Singapore, Singapore, ${ }^{3}$ School of Biological \\ Sciences, Nanyang Technological University, Singapore, Singapore, ${ }^{4}$ School of Biotechnology and Biomolecular Sciences, \\ Centre for Marine Bio-Innovation, University of New South Wales, Sydney, NSW, Australia, ${ }^{5}$ School of Biological, Earth and \\ Environmental Sciences, Centre for Marine Bio-Innovation, University of New South Wales, Sydney, NSW, Australia
}

Pseudomonas aeruginosa PAO1 produces three polysaccharides, alginate, Psl, and Pel that play distinct roles in attachment and biofilm formation for monospecies biofilms. Considerably less is known about their role in the development of mixed species biofilm communities. This study has investigated the roles of alginate, Psl, and Pel during biofilm formation of $P$. aeruginosa in a defined and experimentally informative mixed species biofilm community, consisting of $P$. aeruginosa, Pseudomonas protegens, and Klebsiella pneumoniae. Loss of the Psl polysaccharide had the biggest impact on the integration of $P$. aeruginosa in the mixed species biofilms, where the percent composition of the ps/ mutant was significantly lower (0.06\%) than its wild-type (WT) parent (2.44\%). In contrast, loss of the Pel polysaccharide had no impact on mixed species biofilm development. Loss of alginate or its overproduction resulted in $P$. aeruginosa representing 8.4 and $18.11 \%$, respectively, of the mixed species biofilm. Dual species biofilms of $P$. aeruginosa and $K$. pneumoniae were not affected by loss of alginate, $\mathrm{Pel}$, or $\mathrm{Psl}$, while the mucoid $P$. aeruginosa strain achieved a greater biomass than its parent strain. When $P$. aeruginosa was grown with $P$. protegens, loss of the Pel or alginate polysaccharides resulted in biofilms that were not significantly different from biofilms formed by the WT PAO1. In contrast, overproduction of alginate resulted in biofilms that were comprised of $35-40 \%$ of $P$. aeruginosa, which was significantly higher than the WT (5-20\%). Loss of the Psl polysaccharide significantly reduced the percentage composition of $P$. aeruginosa in dual species biofilms with $P$. protegens $(<1 \%)$. Loss of the Psl polysaccharide significantly disrupted the communal stress resistance of the three species biofilms. Thus, the polysaccharide composition of an individual species significantly impacts mixed species biofilm development and the emergent properties of such communities.

Keywords: exopolysaccharides, biofilms, mixed species consortia, interspecies competition, stress tolerance 


\section{Introduction}

Bacteria predominantly occur as biofilms in the environment and biofilm formation is linked to increased tolerance of bacteria to a range of environmental and host related stressors. As a consequence, considerable experimental effort to understand how bacteria regulate biofilm formation and what effectors are involved in the increased resilience of biofilms has been made. Resistance of biofilm cells has been linked in part to the physiological status of the cells, where gradients of nutrients result in a stratified population of cells. Under these conditions the cells within microcolonies are less active or express stationary phase like responses (Hentzer et al., 2005; Waite et al., 2005). Biofilm formation also occurs in response to regulatory processes including quorum sensing or to exposure to stressors such as sublethal doses of antibiotics and detergents (Whiteley et al., 2001; Folsom et al., 2010).

One of the defining features of the biofilm is the presence of a self-produced extra-cellular matrix. This matrix not only provides the scaffold for adhesion to surfaces and cohesion between cells, but also protects the cells from stresses such as desiccation, oxidizing agents and host immune defenses (DeVault et al., 1990; Ophir and Gutnick, 1994; Pier et al., 2001; O’Toole, 2003; Parsek and Singh, 2003; Friedman and Kolter, 2004; Jackson et al., 2004; Ryder et al., 2007). The matrix can additionally sequester valuable enzymes and nutrients, cellto-cell communication signals and fosters the exchange of genetic material (Stoodley et al., 2002). The matrix is typically comprised of a combination of proteins, extracellular DNA and polysaccharides.

The biofilm matrix of $P$. areuginosa PAO1 has been shown to include at least three polysaccharides, alginate, Psl, and Pel polysaccharides, and their roles during biofilm development have been demonstrated in biofilm populations (single species systems; Colvin et al., 2011, 2012; Ghafoor et al., 2011; Billings et al., 2013; Zhao et al., 2013). Alginate deficient mutants develop biofilms with a decreased proportion of viable cells and contain significantly more extracellular DNA (Ghafoor et al., 2011). It has also been shown that exposure to oxidative stress induces the overproduction of alginate, which protects the biofilms from oxidative radicals (Mathee et al., 1999; Hentzer et al., 2001). Biofilms of psl or alginate deletion mutants failed to form the characteristic mushroom like structures, suggesting these polysaccharides are important for structural development (Ghafoor et al., 2011). Pel was described as being essential for the formation of biofilms by Pseudomonas aeruginosa at the air-liquid interface in static broth cultures (Friedman and Kolter, 2004). Psl also plays an important role in the initiation of biofilm formation (Friedman and Kolter, 2004; Jackson et al., 2004; Matsukawa and Greenberg, 2004; Campisano et al., 2006; Ma et al., 2006). More recently, the visco-elastic properties of Pel and Psl were described (Chew et al., 2014), where it was shown that Psl demonstrated properties consistent with elastic materials, suggesting that it is stiff or rigid. In contrast, the Pel polysaccharide was more viscous and was responsible for the formation of biofilm streamers. These properties have been shown to have important outcomes for biofilms that form in industrial settings. For example, biofilms that lack the Psl polysaccharide showed a reduced tendency to inhibit reverse osmosis membrane performance, suggesting that the strong, cohesive properties of Psl were necessary to make an impermeable biofilm (Barnes et al., 2014). Collectively, these data demonstrate that the individual polysaccharide components of the EPS play important roles in biofilm formation and structure development.

While the roles of the matrix components have been well studied in the context of monospecies biofilm development, considerably less is understood about the roles of the matrix in the development of mixed species biofilm communities. This is particularly relevant because in nature, most biofilms are represented by diverse communities rather than populations of single species. For these mixed species biofilm communities, the organization of the different species may be important for community function and therefore, the matrix potentially plays a vital role in the structural organization of mixed species communities. Experiments investigating dual species biofilms formed by $P$. aeruginosa and Staphylococcus aureus indicated that the production of Pel and Psl were important for the two bacteria to form biofilms together, suggesting that polysaccharide production may be a key factor in community assembly (Billings et al., 2013; Chew et al., 2014). We have recently established a mixed species biofilm community that results in increased overall biomass of the community relative to single species biofilms formed separately by its members (Lee et al., 2014). Further, the mixed species biofilm demonstrated community level stress protection, which was extended to all of the community members, despite some of those members being individually sensitive to those stresses. The mechanisms that drive community assembly and resistance are currently unknown.

In the present study, we have investigated the role of polysaccharides produced by PAO1 in the establishment of a biofilm community, consisting of $P$. aeruginosa PAO1, $P$. protegens Pf-5, and Klebsiella pneumoniae KP-1. Specifically, mutants of $P$. aeruginosa that were deficient in the production of alginate, Pel, and Psl or that over expressed alginate, were compared for the formation of mixed species communities. The results demonstrate that the composition of the mixed species biofilm community was strongly influenced by the ability of $P$. aeruginosa to produce the Psl polysaccharide. This highlights the importance of specific polysaccharides in biofilm community assembly and function.

\section{Materials and Methods}

\section{Bacterial Strains and Culture Media}

Bacteria (Table 1) were routinely cultured in either M9 minimal medium (48 mM Na $2 \mathrm{HPO}_{4} ; 22 \mathrm{mM} \mathrm{KH}_{2} \mathrm{PO}_{4} ; 9 \mathrm{mM} \mathrm{NaCl}$; $19 \mathrm{mM} \mathrm{NH}_{4} \mathrm{Cl} ; 2 \mathrm{mM} \mathrm{MgSO}_{4} ; 0.1 \mathrm{mM} \mathrm{CaCl}_{2}$; and $2 \mathrm{mM}$ glucose) supplemented with $0.2 \% \mathrm{w} / \mathrm{v}$ CAA (supplemented M9 minimal medium), Luria Bertani broth $\left(\mathrm{LB}_{10} ; 10 \mathrm{~g} \mathrm{~L}^{-1} \mathrm{NaCl} ; 10 \mathrm{~g} \mathrm{~L}^{-1}\right.$ tryptone; $5 \mathrm{~g} \mathrm{~L}^{-1}$ yeast extract) or Super Optimal Broth (SOB; 
TABLE 1 | List of bacterial strains used.

\begin{tabular}{|c|c|c|}
\hline Species and strain & Genotypic and phenotypic characteristics ${ }^{4}$ & Source \\
\hline $\begin{array}{l}\text { Pseudomonas aeruginosa } \\
\text { PAO1 }\end{array}$ & & Lee et al. (2014) \\
\hline $\mathrm{PAO} 1 \Delta a l g$ & Isogenic alg8 deletion mutant & Ghafoor et al. (2011) \\
\hline $\mathrm{PAO} 1 \Delta \mathrm{pel}$ & Isogenic pelF deletion mutant & Ghafoor et al. (2011) \\
\hline $\mathrm{PAO} 1 \Delta p s /$ & Isogenic pslA deletion mutant & Ghafoor et al. (2011) \\
\hline PDO300 $\triangle m u c A$ & Mutation in the mucA22 allele & Mathee et al. (1999) \\
\hline PAO1-eYFP & $\begin{array}{l}\text { Carries the gene encoding eYFP in the intergenic region between coding region of } \mathrm{g} / \mathrm{mS} \\
\text { and its downstream gene; } \mathrm{Gm}^{\mathrm{R}}\end{array}$ & Lee et al. (2014) \\
\hline PAO1 $\Delta a l g$-eYFP & & This project \\
\hline PAO1 1 pel-eYFP & & This project \\
\hline $\mathrm{PAO} 1 \Delta p s /-e Y F P$ & & This project \\
\hline PDO300 $\Delta m u c A-e Y F P$ & & This project \\
\hline${ }^{1} P$. protegens Pf-5 & & Lee et al. (2014) \\
\hline Pf-5-eCFP & $\begin{array}{l}\text { Carries the gene encoding eCFP in the intergenic region between coding region of } \mathrm{g} / \mathrm{mS} \\
\text { and its downstream gene; } \mathrm{Gm}^{\mathrm{R}}\end{array}$ & Lee et al. (2014) \\
\hline $\begin{array}{l}{ }^{2} \text { Klebsiella pneumoniae } \\
\text { KP-1 }\end{array}$ & & Lee et al. (2014) \\
\hline KP-1 -DsRed & $\begin{array}{l}\text { Carries the gene encoding DsRedExpress in the intergenic region between coding } \\
\text { region of } g / m S \text { and its downstream gene; } \mathrm{Gm}^{\mathrm{R}}\end{array}$ & Lee et al. (2014) \\
\hline \multicolumn{3}{|l|}{ Escherichia coli } \\
\hline JM109 & 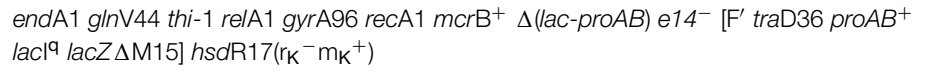 & Yanisch-Perron et al. (1985) \\
\hline HPS1 & $\mathrm{F}^{-} \Delta($ lab-proAB) endA1 gyrA96 hsdR17 supE44 relA1 recA1 thi rif $\mathrm{R}$ zzx::mini-Tn5Lac4 & Choi et al. (2005) \\
\hline CC118 $\lambda$ pir & $\Delta($ ara-leu $)$ araD $\Delta$ lacX74 galE ga/K phoA20 thi-1 rpsE rpoB argE(Am) recAl $\lambda$ pir & Choi et al. (2005) \\
\hline $\mathrm{DH} 5 \alpha \lambda$ pir & $\begin{array}{l}\mathrm{F}^{-}, \Phi 80 \mathrm{~d} / a c Z \Delta \mathrm{M} 15 \Delta\left(\text { (lacZYA-argF)U169 deoR recA1 endA1 } h s d \mathrm{R} 17\left(\mathrm{r}_{\mathrm{K}}{ }^{-}, \mathrm{m}_{\mathrm{K}}{ }^{+}\right) \text {phoA }\right. \\
\text { supE44 thi-1 } \lambda \text { pir }\end{array}$ & Choi et al. (2005) \\
\hline S17-1 $\lambda$ pir & hsdR recA pro RP4-2 (Tc::Mu; Km::Tn7; $\lambda$ pir) & Miller and Mekalanos (1988) \\
\hline HB101 & $\begin{array}{l}\mathrm{F}^{-}, \text {hsdS20 }\left(\mathrm{rb}^{-}, \mathrm{mb}^{-}\right), \text {supE44, ara14, ga/K2, lacY1, proA2, } \\
\text { rpsL20 }\left(\mathrm{Str}^{\mathrm{R}}\right), x y l-5, \mathrm{mtl}^{-1}, \mathrm{l-}, \text { recA13, } \text { mcrA }^{-}, \text {mcrB }^{-}\end{array}$ & Lambertsen et al. (2004) \\
\hline
\end{tabular}

${ }_{1}^{1}$ Pseudomonas fluorescens Pf-5 has recently been renamed as P. protegens Pf-5 (Ramette et al., 2011; Lim et al., 2013)

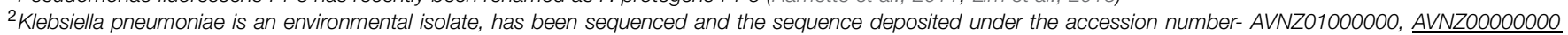
(Lee et al., 2013).

$\mathrm{Gm}^{\mathrm{R}}$, Gentamicin resistance; $\mathrm{Str}^{\mathrm{R}}$, Streptomycin resistance.

$10 \mathrm{mM} \mathrm{NaCl} ; 2.5 \mathrm{mM} \mathrm{KCl} ; 10 \mathrm{mM} \mathrm{MgCl}_{2} ; 10 \mathrm{mM} \mathrm{MgSO}_{4} ; 20 \mathrm{~g}$ $\mathrm{L}^{-1}$ tryptone; $5 \mathrm{~g} \mathrm{~L}^{-1}$ yeast extract).

\section{Transformation of $P$. aeruginosa EPS Mutants by Electroporation}

Electrocompetent $P$. aeruginosa EPS mutants $\triangle m u c A, \Delta a l g$, $\Delta p e l$, and $\Delta p s l$ were prepared as described (Choi et al., 2006). During transformation, the ColE1 replicon-based delivery plasmid and the helper plasmid, pTNS1 (Table 2), were added to the electrocompetent cells and electroporated $(25 \mu \mathrm{F}, 200 \Omega$ and $2.5 \mathrm{kV} \mathrm{cm}^{-1}$ ) using a Gene Pulser ${ }^{\mathrm{TM}}$ apparatus (BIO-RAD, USA). Transformed cells were recovered by the addition of ice cold Super Optimal Broth with Catabolite repression (SOC; SOB supplemented with $2 \% \mathrm{w} / \mathrm{v}$ glucose) and incubated with shaking for $3 \mathrm{~h}$ at $37^{\circ} \mathrm{C}$. Recovered cells were plated onto $\mathrm{LB}_{5}$ agar $(5 \mathrm{~g}$ $\mathrm{L}^{-1} \mathrm{NaCl} ; 10 \mathrm{~g} \mathrm{~L}^{-1}$ tryptone; $5 \mathrm{~g} \mathrm{~L}^{-1}$ yeast extract; $1.5 \% \mathrm{w} / \mathrm{v}$ agar) plates were supplemented with $100 \mu \mathrm{g} \mathrm{mL}^{-1}$ gentamicin for the selection of transformants.

\section{Determination of $\mathrm{Tn} 7$ Insertion Site}

Colony PCR was used to verify chromosomal Tn7 insertion using primers specific for the insertion site (Table 3) using a $\mathrm{C} 1000^{\mathrm{TM}}$ thermal cycler (BIO-RAD, USA) with an initial denaturation at $97^{\circ} \mathrm{C}$ for $3 \mathrm{~min}$ followed by 35 cycles of amplification (denaturation at $97^{\circ} \mathrm{C}, 30 \mathrm{~s}$; annealing at $55^{\circ} \mathrm{C}, 30 \mathrm{~s}$; extension at $72^{\circ} \mathrm{C}, 1 \mathrm{~min}$ ) and a final extension at $72^{\circ} \mathrm{C}$ for $10 \mathrm{~min}$. The PCR product was visualized on a $1 \% \mathrm{w} / \mathrm{v}$ agarose gel and sequenced.

\section{Flow Cells Dynamics Experiments}

Biofilms were cultivated in three-channel flow cells (channel dimensions, $1 \mathrm{~mm} \times 4 \mathrm{~mm} \times 40 \mathrm{~mm}$; Biocentrum-DTU; Sternberg and Tolker-Nielsen, 2006). The flow cells were supplied with supplemented M9 minimal medium at $9 \mathrm{~mL} \mathrm{~h}^{-1}$ (mean velocity $=0.625 \mathrm{~mm} \mathrm{~s}^{-1}$ ) with a Reynolds number of 1.12. Each channel was injected with $0.5 \mathrm{~mL}$ of diluted overnight culture containing approximately $1 \times 10^{8} \mathrm{cfu} \mathrm{mL}^{-1}$. Mixed species biofilms were established by inoculating mixed cultures of PAO1 EPS mutants, Pf-5, and KP-1 in the ratio of 5:5:1, respectively.

\section{SDS Treatment}

Flow cells biofilms were grown in M9 supplemented with $2 \mathrm{mM}$ glucose and $0.2 \% \mathrm{w} / \mathrm{v}$ CAA. After 3 days, biofilms were treated with M9 glucose, CAA, and $0.1 \%$ SDS under flow conditions for 
TABLE 2 | List of plasmids used in this study.

\begin{tabular}{|c|c|c|}
\hline Plasmid & Relevant characteristic ${ }^{3}$ & Source \\
\hline pTNS1 & Helper plasmid, providing the $T n 7$ transposition function. $A p^{R}$, R6K ori, ori $T$ & Choi et al. (2005) \\
\hline pTNS2 & Helper plasmid, providing the $T n 7$ transposition function. $A^{R}{ }^{R}, R 6 K$ ori, ori $T$ & AY884833 1,2 \\
\hline pTNS2-ColE1 & Helper plasmid, providing the $T n 7$ transposition function. $A p^{R}$, ColE1 ori, ori $T$ & Lee et al. (2014) \\
\hline $\begin{array}{l}\text { pUC18T- } \\
\text { mini-Tn7T-Gm-eYFP/HPS1 }\end{array}$ & pUC18 -based delivery plasmid for mini-Tn7-Gm-eYFP. Ap ${ }^{R}, \mathrm{Gm}^{\mathrm{R}}$, ColE1 ori, oriT & DQ493879 1,2 \\
\hline pUC18TR6K- mini-Tn7T-Gm-eYFP & pUC18 -based delivery plasmid for mini-Tn7-Gm-eYFP. Ap ${ }^{R}, G m^{R}$, R6K ori, oriT & Lee et al. (2014) \\
\hline pRK600 & Mobilizing plasmid, providing the mobilization ability during conjugation. $\mathrm{Ap}^{\mathrm{R}}, \mathrm{Cm}^{\mathrm{R}}, \mathrm{R} 6 \mathrm{~K}$ ori & Laboratory stock \\
\hline pUC18TR6K-mini-Tn7T & $\begin{array}{l}\text { pUC18 -based vector plasmid for construction of R6K replicon-based delivery plasmids in this } \\
\text { project. } \mathrm{Ap}^{\mathrm{R}}, \mathrm{R} 6 \mathrm{~K} \text { ori, oriT }\end{array}$ & AY712953² \\
\hline
\end{tabular}

${ }^{1}$ Plasmids were generously provided by Herbert P. Schweizer (Choi et al., 2005).

${ }^{2}$ National Center for Biotechnology Information (NCBI) accession number.

${ }^{3} A p^{R}$, Ampicillin resistance; $\mathrm{Cm}^{\mathrm{R}}$, Chloramphenicol resistance; $\mathrm{Gm}^{\mathrm{R}}$, Gentamicin resistance.

TABLE 3 | List of primers used.

\begin{tabular}{|c|c|c|}
\hline Primer & Sequence & Description \\
\hline ColE1_F & 5'AGGATCCCCGGGGATAACGCAGGAAAGAACAT3' & $\begin{array}{l}\text { Primer is used during PCR amplification of ColE1 ori. Primer is flanked } \\
\text { with Smal site at } 5^{\prime} \text { end. }\end{array}$ \\
\hline ColE1_R & 5'GATTACGAATTCCTGTCAGACCAAGTTACTC3' & $\begin{array}{l}\text { Primer is used during PCR amplification of ColE1 ori. Primer is flanked } \\
\text { with EcoRI site at } 5^{\prime} \text { end. }\end{array}$ \\
\hline $\operatorname{Tn} 7 \mathrm{R}$ & 5'CAGCATAACTGGACTGATTTCAG3' & Common primer used for checking chromosomal insertion of Tn7. \\
\hline PAglmS-down & 5'GCACATCGGCGACGTGCTCTC3' & Primer used with Tn7R to check chromosomal insertion of Tn7 in PAO1 \\
\hline
\end{tabular}

$2 \mathrm{~h}$. Images were collected before and after the treatment for the quantification of biomass.

\section{Microscopy, Image and Statistical Analysis}

All microscopic observations and image acquisition were performed using a CLSM (LSM 780, Carl Zeiss, Germany). For each channel, five image stacks were acquired, covering a total area of approximately $9 \times 10^{5} \mu \mathrm{m}^{2}$, which was more than the suggested minimum of $1 \times 10^{5} \mu \mathrm{m}^{2}$ to acquire representative data (Korber et al., 1993). For image analysis, a total of 15 image stacks (five from each experiment) were quantified for each biofilm type using IMARIS (Bitplane AG, Switzerland). Statistical analysis was performed using Graph pad PRISM.

\section{Results}

\section{The Role of Pel, Psl, and Alginate in the Development of Three-Species Biofilm Communities}

To determine the roles of alginate, Psl, and Pel produced by $P$. aeruginosa in mixed species biofilm community development, polysaccharide mutants of $P$. aeruginosa, alg, mucA, pel, and $p s l$ were cultivated with $P$. protegens and $K$. pneumoniae as triple species biofilms. Initial attachment of the alginate overproducing strain, $m u c A$, was similar to the wild-type (WT) P. aeruginosa (Figure 1; Supplementary Figure S1). However, in contrast to the WT $P$. aeruginosa, the biovolume of the mucA mutant remained constant at $20 \%$ throughout the duration of the experiment, which was significantly higher than the WT (2\%). Mutants in the alg and pel polysaccharide genes showed an increase in the amount of $P$. aeruginosa present in the three species biofilm community during the initiation of biofilm formation (Figures 1B,D,F). When the alg mutant was included in the biofilm, the architecture of $P$. protegens changed from one dominated by microcolonies to a more filamentous biofilm and the alg mutant completely covered the top of the biofilm at day 7 (Supplementary Figure S1). In contrast, the psl mutant was below the detection level in the triple species biofilms (Figures 1E,F) with $P$. protegens and $K$. pneumoniae accounting for 52.24 and $47.69 \%$ of the biofilm biomass, respectively (Figure 1E).

\section{The Role of Pel, Psl and Alginate in Dual Species Biofilm Development}

Similarly, the roles of the $P$. aeruginosa polysaccharides in mediating dual species biofilm interactions were also investigated. When grown as a dual species biofilm with P. protegens (Figure 2; Supplementary Figure S2) the mucA mutant showed a significant increase $(35-40 \%)$ in relative biovolume compared to the WT $P$. aeruginosa (5-20\%). There was no significant difference in the biovolumes for the pel and alg mutants relative to the WT. As observed for the three species biofilm, the psl mutant ( $<1 \%$ biovolume) was also severely impaired in its ability to establish a dual species biofilm with P. protegens.

When the polysaccharide mutants formed dual species biofilms with K. pneumoniae (Figure 3), alg and pel mutants exhibited significant increases at day 1 , but not for the remainder of the experiment, relative to the WT $P$. aerugionsa. There 


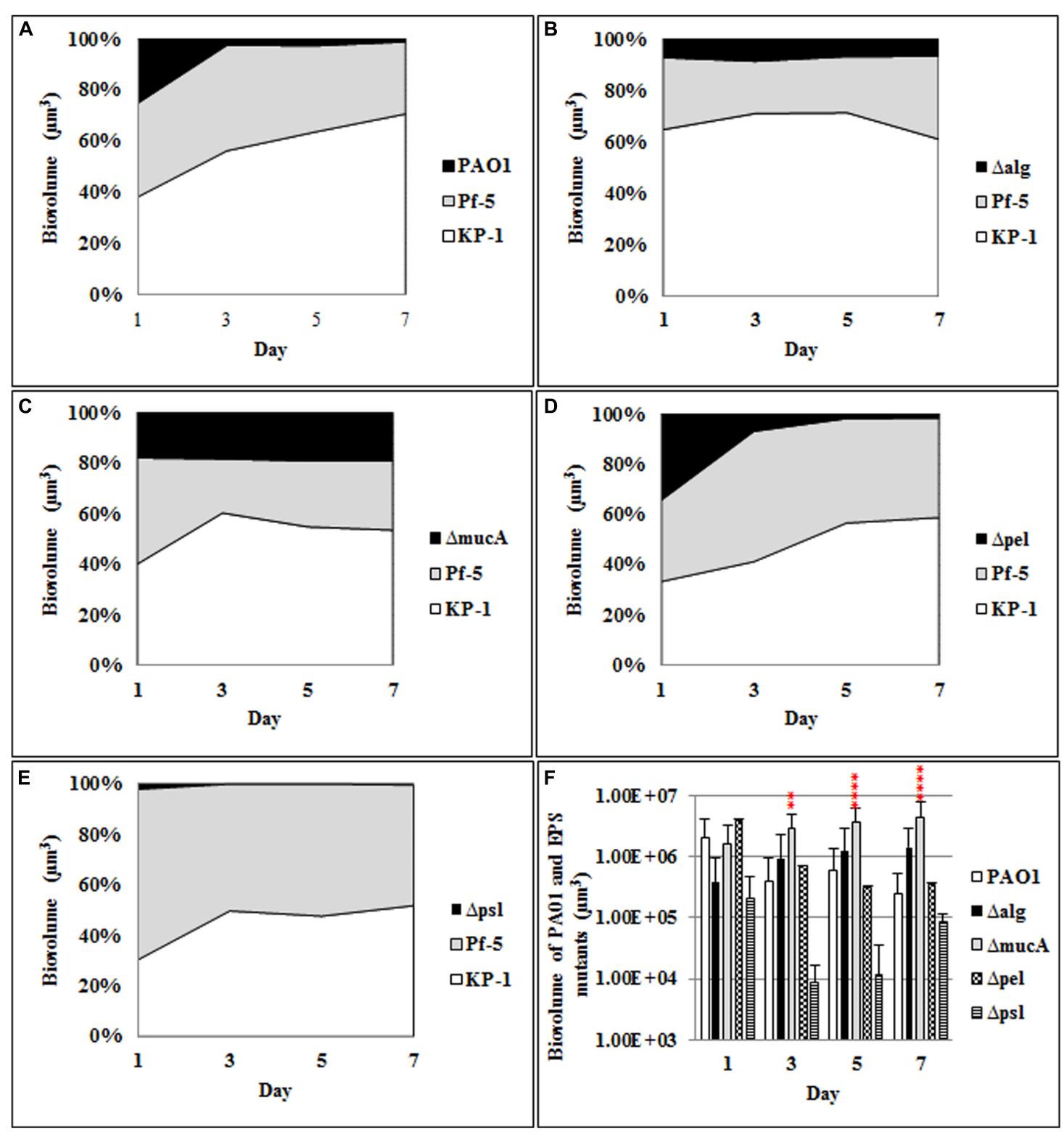

FIGURE 1 | Spatial and temporal development of Pseudomonas aeruginosa polysaccharide mutants grown with $P$. protegens and Klebsiella pneumoniae as three species biofilms. The proportion of the three species within the mixed species over the 7 days period was determined by quantitative image analysis. (A) $P$. aeruginosa wild-type (WT), (B) $\Delta$ alg, (C) $\triangle m u c A,(\mathbf{D}) \Delta p e l,(\mathbf{E}) \Delta p s l$, and (F) biovolumes of $P$. aeruginosa $W T$ and polysaccharide mutants. Statistical analysis was performed vs. the corresponding WT samples grown in parallel ${ }^{*} P<0.01,{ }^{* * * *} P<0.0001$. was a statistically significant increase in the biofilm biomass of the alginate over producing strain, $m u c A$, for days 3-7 of biofilm development (39-47\%) relative to the WT P. aeruginosa (16-30\%).

The data suggest that the mucoid strain of $P$. aeruginosa is better able to compete in mixed species biofilm communities while the $p s l$ mutant is generally less fit under these conditions. The primary changes in $P$. aeruginosa biofilm biomass were observed when it was grown with $P$. protegens suggesting the resource competition in the mixed species biofilms is strongest between these two closely related species.

\section{The Role of Polysaccharides in the Stress Resistance of Mixed Species Biofilms}

It was previously shown that this mixed species biofilm community displays enhanced resistance to SDS and antibiotic stress relative to biofilms formed by the individual species alone (Lee et al., 2014). Further, the stress resistance was a communal property, where all three species were equally protected, despite monospecies biofilms of $P$. protegens being highly sensitive to SDS exposure. To determine the role of the polysaccharide component of the EPS in stress resistance of mixed species biofilms, mutants that either overproduce alginate, $m u c A$, or that 

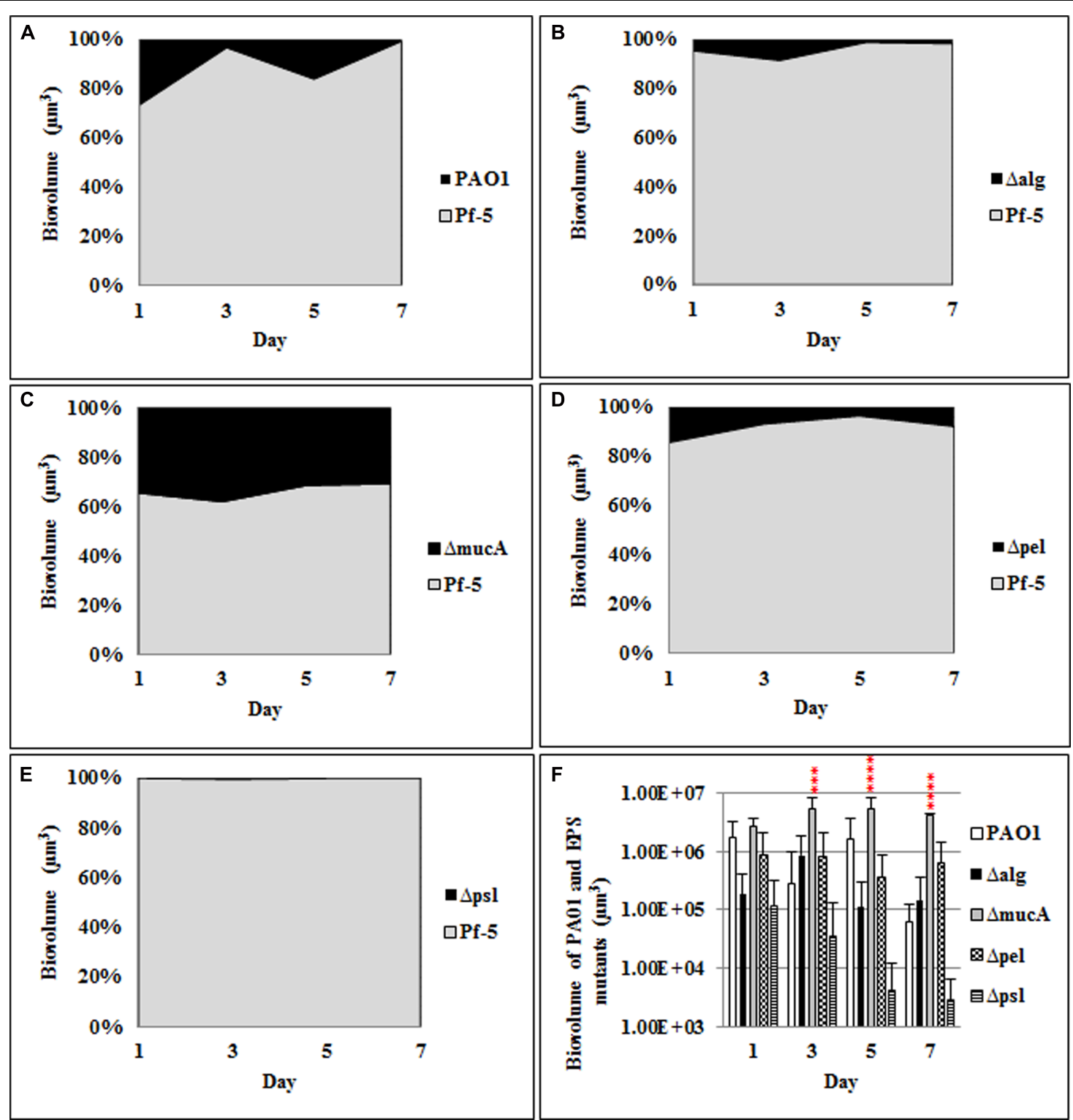

FIGURE 2 | Spatial and temporal development of $\boldsymbol{P}$ aeruginosa polysaccharide mutants grown with $\boldsymbol{P}$. protegens as dual species biofilms. The proportion of the two species was calculated by quantitative image analysis. (A) $P$. aeruginosa WT, (B) $\Delta a l g$, (C) $\Delta m u c A$,
(D) $\Delta p e l$, (E) $\Delta p s l$, and (F) biovolumes of $P$. aeruginosa and polysaccharide mutants. Statistical analysis was performed vs. the corresponding WT samples grown in parallel, which were very similar in all cases ${ }^{* * *} P<0.001,{ }^{* * * *} P<0.0001$. were defective for the production of Psl were tested for their contribution to the SDS resistance of the three species biofilms. These two strains were used since the mucA strain showed an increased proportion in the mixed species biofilm, while the psl mutant was less competitive during mixed species growth. Mixed species biofilms formed with the mucA mutant showed similar protection as the WT $P$. aeruginosa and protection was shared across all three species (Figure 4). In contrast, mixed species biofilms that included the $p s l$ mutant showed a significant reduction in biofilm biomass after SDS stress. The biomass of $P$. protegens was reduced by fivefold, indicating that it was no longer protected during mixed species biofilm growth. The biomass of K. pneumoniae showed similar amounts of biofilm before and after surfactant exposure and hence was unaffected by the change in biofilm composition.

\section{Discussion}

The dynamics of biofilm formation are influenced by a number of biotic and abiotic factors (Costerton et al., 1994; Wolfaardt et al., 1994). While the effects of polysaccharides on biofilm development have been well studied for biofilm populations, less is understood about their role during the development of 


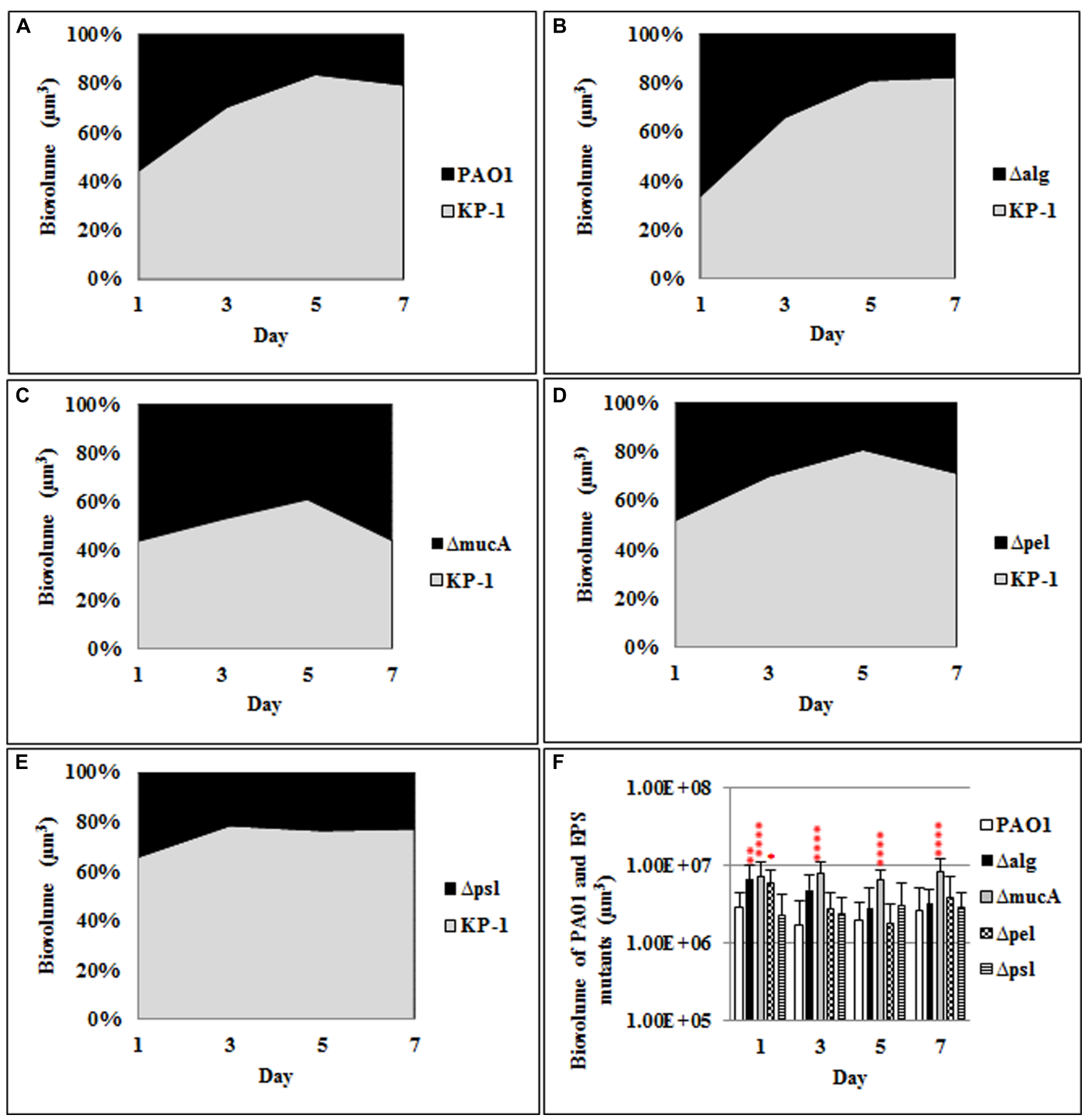

FIGURE 3 | Spatial and temporal development of $\boldsymbol{P}$. aeruginosa polysaccharide mutants grown with $K$. pneumoniae as dual species biofilms. The proportion of the two species was calculated by quantitative image analysis. (A) P. aeruginosa WT, (B) $\Delta a l g$, (C) $\Delta m u c A$, (D) $\Delta p e l$, (E)
$\Delta p s l$, and (F) biovolumes of $P$. aeruginosa and polysaccharide mutants. Statistical analysis was performed vs. the corresponding WT samples grown in parallel, which were very similar in all cases ${ }^{*} P<0.01,{ }^{*} P<0.001$, $* * * * P<0.0001$ mixed species biofilm communities. We have investigated here the contribution of the three known polysaccharides produced by $P$. aerugionsa to determine their role in mixed species biofilm development. It was observed that the initial attachment of the mucoid $P$. aerugionsa mucA mutant was higher than for the WT, as evidenced by the increased proportion of the mutant in the mixed species biofilm at day 1 and for the remainder of the biofilm development cycle. This effect was seen when the $m u c A$ strain was present in the three species as well as dual species biofilms. Alginate over expression in $P$. aerugionsa is frequently associated with chronic lung infections and mucoid strains have been shown to have increased resistance to stressors. Here we observed that alginate over production resulted in an increase in the biofilm biomass of $P$. aeruginosa relative to $P$. protegenes and K. pneumoniae. Therefore, over production of alginate could enhance the competitive fitness of $P$. aerugionsa during mixed species biofilm formation during chronic lung infection.

The pel mutant was similar to the WT in its contribution to the biofilm and thus, under these conditions, may play a lesser role in mixed species biofilm development. While Pel was not essential, loss of this polysaccharide resulted in alteration of the 


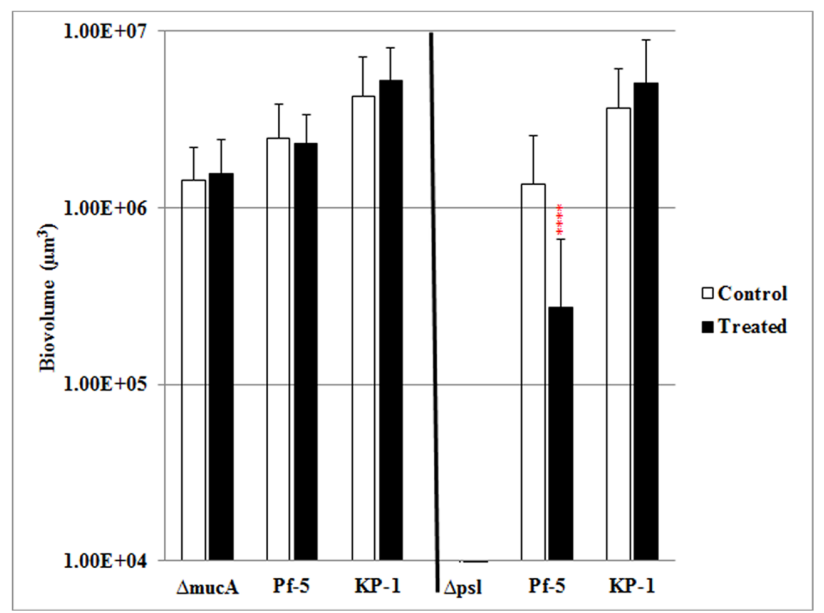

FIGURE 4 | The role of alginate and Psl in stress resistance of mixed species biofilms. Three species biofilms were formed for 4 days and exposed to $0.1 \%$ SDS for $2 \mathrm{~h}$. The biofilm biovolumes of $P$. aeruginosa mucA (left), ps/ (right), Pf-5, and KP-1 were determined by quantitative image analysis before and after SDS treatment. Statistical analysis was performed vs. the corresponding WT samples grown in parallel, which were very similar in all cases $* * * * P<0.0001$.

biofilm structure. It was observed that the height of microcolonies formed by the pel mutant ranged from 40 to $50 \mu \mathrm{m}$ compared to the WT microcolonies, for which the microcolony heights ranged from 70 to $80 \mu \mathrm{m}$ (data not shown). It has also been shown that loss of Pel from $P$. aeruginosa biofilms results in stiffer, more rigid biofilms (Chew et al., 2014). Therefore, the loss of Pel may stiffen the mixed species biofilm, preventing the expansion of microcolony formation. In dual species biofilms of $P$. aeruginosa and $S$. aureus, Pel was shown to be essential for the two species to form mixed, integrated biofilm communities (Chew et al., 2014).

In contrast to the pel mutant, the psl mutant was almost completely excluded from both triple and dual $(P$. aeruginosa $+P$. protegens $)$ mixed species biofilms. This observation is in agreement with the role of Psl in monospecies biofilm formation, where loss of the polysaccharide results in a severe defect in biofilm formation (Ghafoor et al., 2011). During attachment, Psl is anchored on the cell surface in a helical pattern, which promotes cell-cell interactions and assembly of a matrix, to hold the bacteria in the biofilm and on the surface (Ma et al., 2009). When grown with S. aureus, the Psl mutant formed well mixed dual species biofilms (Chew et al., 2014), further supporting the role of Psl as a rigid polymer responsible for the formation of stiff, inflexible microcolonies.

Given that mixed species biofilms display enhanced stress resistance (Lee et al., 2014) and that the polysaccharide Psl has been shown to play a role in the protection of other species in biofilm communities, the roles of alginate and Psl in the surfactant stress response of mixed species biofilms were tested. Previously, it was shown that monospecies biofilms of $P$. protegens were sensitive to SDS stress, but when $P$. protegens was grown as a biofilm with $P$. aeruginosa and $K$. pneumoniae, it was protected in the mixed species biofilm. When the WT $P$. aeruginosa was replaced with the $m u c A$ mutant, the all of the community members were equally protected during mixed species biofilm growth. In contrast, when the mixed species biofilm included the psl mutant, the protection was lost and both $P$. aeruginosa and $P$. protegens, showed a significant decrease after exposure to SDS stress. This observation suggests that Psl is required for community level protection against SDS stress. Similarly, it was previously shown that Psl plays a role in mediating antibiotic resistance of $P$. aeruginosa biofilms and that the antibiotic resistance afforded by Psl could also protect Escherichia coli and S. aureus when grown as co-culture biofilms with $P$. aeruginosa (Billings et al., 2013). Thus, Psl may play a more general role in mediating stress tolerance of mono and mixed species biofilms, hence providing protection of biofilm populations as well as communities.

\section{Conclusion}

The data presented here show that specific polysaccharides, such as Psl and alginate play important roles for $P$. aeruginosa during mixed species biofilm growth. The production of these polysaccharides not only impact the competitive fitness of a species during mixed biofilm growth, but also has significant effects on the function of that community. Therefore, biofilm matrix biomolecules may individually play significant roles in the formation of biofilm communities, arguably the natural state of most biofilm systems, and these functions may not be evident from population based studies.

\section{Acknowledgments}

The authors would like to acknowledge financial support from the Singapore Centre on Environmental Life Sciences Engineering (SCELSE), whose research is supported by the National Research Foundation Singapore, Ministry of Education, Nanyang Technological University and National University of Singapore, under its Research Centre of Excellence Program.

\section{Supplementary Material}

The Supplementary Material for this article can be found online at: http://journal.frontiersin.org/article/10.3389/fmicb. 2015.00851

FIGURE S1 | Ortho views of confocal micrographs of mixed species biofilms composed of Pseudomonas aeruginosa polysaccharide mutants (yellow), P. protegens (blue) and Klebsiella pneumoniae (red) grown on M9 supplemented with $2 \mathbf{m M}$ glucose $+\mathbf{0 . 2} \%$ CAA. (A) $P$. aeruginosa wild-type (WT), (B) $\Delta a l g,(C) \Delta m u c A$, (D) $\Delta$ pel, and (E) $\Delta p s l$. The top and side images of each panel represent the $x-z$ and $y-z$ planes, respectively. The green and red lines indicate the positions corresponding to the $x-z$ and $y-z$ cross sections, respectively. The blue line indicates the $x-y$ plane of the main panel. Magnification 200x. 
FIGURE S2 | Dual species biofilms comprised of $P$. aeruginosa polysaccharide EPS mutants and $P$. protegens grown on $2 \mathrm{mM}$ glucose $+\mathbf{0 . 2} \%$ CAA. The ortho view of confocal micrographs of dual species composed of $P$. aeruginosa polysaccharide mutants (yellow) and $P$. protegens (blue) imaged over 7 days. The top and side images of each panel represent the $x-z$ and $y-z$ planes, respectively. The green and red lines indicate the positions corresponding to the $x-z$ and $y-z$ cross sections, respectively. The blue line indicates the $x-y$ plane of the main panel. Magnification $200 x$.

\section{References}

Barnes, R. J., Bandi, R. R., Chua, F., Low, J. H., Aung, T., Barraud, N., et al. (2014). The roles of Pseudomonas aeruginosa extracellular polysaccharides in biofouling of reverse osmosis membranes and nitric oxide induced dispersal. J. Mem. Sci. 466, 161-172. doi: 10.1016/j.memsci.2014.04.046

Billings, N., Millan, M., Caldara, M., Rusconi, R., Tarasova, Y., Stocker, R., et al. (2013). The extracellular matrix component Psl provides fast-acting antibiotic defense in Pseudomonas aeruginosa biofilms. PLoS Pathog. 9:e1003526. doi: 10.1371/journal.ppat.1003526

Campisano, A., Schroeder, C., Schemionek, M., Overhage, J., and Rehm, B. H. (2006). PslD is a secreted protein required for biofilm formation by Pseudomonas aeruginosa. Appl. Environ. Microbiol. 72, 3066-3068. doi: 10.1128/aem.72.4.3066-3068.2006

Chew, S. C., Kundukad, B., Seviour, T., Van Der Maarel, J. R., Yang, L., Rice, S. A., et al. (2014). Dynamic remodeling of microbial biofilms by functionally distinct exopolysaccharides. MBio 5:e01536-14. doi: 10.1128/mBio.01 536- 14

Choi, K. H., Gaynor, J. B., White, K. G., Lopez, C., Bosio, C. M., KarkhoffSchweizer, R. R., et al. (2005). A Tn7-based broad-range bacterial cloning and expression system. Nat. Methods 2, 443-448. doi: 10.1038/nmeth765

Choi, K. H., Kumar, A., and Schweizer, H. P. (2006). A 10-min method for preparation of highly electrocompetent Pseudomonas aeruginosa cells: application for DNA fragment transfer between chromosomes and plasmid transformation. J. Microbiol. Methods 64, 391-397. doi: 10.1016/j.mimet.2005.06.001

Colvin, K. M., Gordon, V. D., Murakami, K., Borlee, B. R., Wozniak, D. J., Wong, G. C., et al. (2011). The pel polysaccharide can serve a structural and protective role in the biofilm matrix of Pseudomonas aeruginosa. PLoS Pathog. 7:e1001264. doi: 10.1371/journal.ppat.1001264

Colvin, K. M., Irie, Y., Tart, C. S., Urbano, R., Whitney, J. C., Ryder, C., et al. (2012). The Pel and Psl polysaccharides provide Pseudomonas aeruginosa structural redundancy within the biofilm matrix. Environ. Microbiol. 14, 1913-1928. doi: 10.1111/j.1462-2920.2011.02657.x

Costerton, J., Lewandowski, Z., Debeer, D., Caldwell, D., Korber, D., and James, G. (1994). Biofilms, the customized microniche. J. Bacteriol. 176, 2137-2142.

DeVault, J. D., Kimbara, K., and Chakrabarty, A. M. (1990). Pulmonary dehydration and infection in cystic fibrosis: evidence that ethanol activates alginate gene expression and induction of mucoidy in Pseudomonas aeruginosa. Mol. Microbiol. 4, 737-745. doi: 10.1111/j.1365-2958.1990.tb 00644.x

Folsom, J., Richards, L., Pitts, B., Roe, F., Ehrlich, G., Parker, A., et al. (2010). Physiology of Pseudomonas aeruginosa in biofilms as revealed by transcriptome analysis. BMC Microbiol. 10:294. doi: 10.1186/1471-2180-10-294

Friedman, L., and Kolter, R. (2004). Genes involved in matrix formation in Pseudomonas aeruginosa PA14 biofilms. Mol. Microbiol. 51, 675-690. doi: 10.1046/j.1365-2958.2003.03877.x

Ghafoor, A., Hay, I. D., and Rehm, B. H. (2011). Role of exopolysaccharides in Pseudomonas aeruginosa biofilm formation and architecture. Appl. Environ. Microbiol. 77, 5238-5246. doi: 10.1128/AEM.00637-11

Hentzer, M., Eberl, L., and Givskov, M. (2005). Transcriptome analysis of Pseudomonas aeruginosa biofilm development: anaerobic respiration and iron limitation. Biofilms 2, 37-61. doi: 10.1017/S14790505050 01699

Hentzer, M., Teitzel, G. M., Balzer, G. J., Heydorn, A., Molin, S., Givskov, M., et al. (2001). Alginate overproduction affects Pseudomonas aeruginosa biofilm structure and function. J. Bacteriol. 183, 5395-5401. doi: 10.1128/JB.183.18.5395-5401.2001
FIGURE S3 | Dual species biofilms of $P$. aeruginosa polysaccharide mutants and $K$. pneuomoniae grown in $2 \mathrm{mM}$ glucose $+0.2 \% \mathrm{CAA}$. The ortho view of confocal micrographs of dual species composed of $P$. aeruginosa polysaccharide mutants (yellow) and K. pneumoniae (red) imaged over 7 days. The top and side images of each panel represent the $x-z$ and $y-z$ planes, respectively. The green and red lines indicate the positions corresponding to the $x-z$ and $y-z$ cross sections, respectively. The blue line indicates the $x-y$ plane of the main panel. Magnification $200 x$.

Jackson, K. D., Starkey, M., Kremer, S., Parsek, M. R., and Wozniak, D. J. (2004). Identification of psl, a locus encoding a potential exopolysaccharide that is essential for Pseudomonas aeruginosa PAO1 biofilm formation. J. Bacteriol. 186, 4466-4475. doi: 10.1128/jb.186.14.4466-4475.2004

Korber, D., Lawrence, J., Hendry, M., and Caldwell, D. (1993). Analysis of spatial variability within $\mathrm{Mot}^{+}$and $\mathrm{Mot}^{-}$Pseudomonas fluorescens biofilms using representative elements. Biofouling 7, 339-358. doi: $10.1080 / 08927019309386264$

Lambertsen, L., Sternberg, C., and Molin, S. (2004). Mini-Tn7 transposons for site-specific tagging of bacteria with fluorescent proteins. Environ. Microbiol. 6, 726-732. doi: 10.1111/j.1462-2920.2004.00605.x

Lee, K. W., Arumugam, K., Purbojati, R. W., Tay, Q. X., Williams, R. B., Kjelleberg, S., et al. (2013). Draft genome sequence of Klebsiella pneumoniae strain KP-1. Gen. Announc. 1:e01082-13. doi: 10.1128/genomeA.01 082-13

Lee, K. W., Periasamy, S., Mukherjee, M., Xie, C., Kjelleberg, S., and Rice, S. A. (2014). Biofilm development and enhanced stress resistance of a model, mixedspecies community biofilm. ISME J. 8, 894-907. doi: 10.1038/ismej.2013.194

Lim, Y. W., Schmieder, R., Haynes, M., Willner, D., Furlan, M., Youle, M., et al. (2013). Metagenomics and metatranscriptomics: windows on CFassociated viral and microbial communities. J. Cyst. Fibros. 12, 154-164. doi: 10.1016/j.jcf.2012.07.009

Ma, L., Conover, M., Lu, H., Parsek, M. R., Bayles, K., and Wozniak, D. J. (2009). Assembly and development of the Pseudomonas aeruginosa biofilm matrix. PLoS Pathog. 5:e1000354. doi: 10.1371/journal.ppat.1000354

Ma, L., Jackson, K. D., Landry, R. M., Parsek, M. R., and Wozniak, D. J. (2006). Analysis of Pseudomonas aeruginosa conditional psl variants reveals roles for the psl polysaccharide in adhesion and maintaining biofilm structure postattachment. J. Bacteriol. 188, 8213-8221. doi: 10.1128/jb.01 202-06

Mathee, K., Ciofu, O., Sternberg, C., Lindum, P. W., Campbell, J. I., Jensen, P., et al. (1999). Mucoid conversion of Pseudomonas aeruginosa by hydrogen peroxide: a mechanism for virulence activation in the cystic fibrosis lung. Microbiology 145, 1349-1357. doi: 10.1099/13500872-145-6-1349

Matsukawa, M., and Greenberg, E. P. (2004). Putative exopolysaccharide synthesis genes influence Pseudomonas aeruginosa biofilm development. J. Bacteriol. 186, 4449-4456. doi: 10.1128/jb.186.14.4449-4456.2004

Miller, V. L., and Mekalanos, J. J. (1988). A novel suicide vector and its use in construction of insertion mutations: osmoregulation of outer membrane proteins and virulence determinants in Vibrio cholerae requires toxR. J. Bacteriol. 170, 2575-2583.

Ophir, T., and Gutnick, D. L. (1994). A role for exopolysaccharides in the protection of microorganisms from desiccation. Appl. Environ. Microbiol. 60, $740-745$.

O’Toole, G. A. (2003). To build a biofilm. J. Bacteriol. 185, 2687-2689. doi: 10.1128/JB.185.9.2687-2689.2003

Parsek, M. R., and Singh, P. K. (2003). Bacterial biofilms: an emerging link to disease pathogenesis. Annu. Rev. Microbiol. 57, 677-701. doi: 10.1146/annurev.micro.57.030502.090720

Pier, G. B., Coleman, F., Grout, M., Franklin, M., and Ohman, D. E. (2001). Role of alginate $\mathrm{O}$ acetylation in resistance of mucoid Pseudomonas aeruginosa to opsonic phagocytosis. Infect. Immun. 69, 1895-1901. doi: 10.1128/iai.69.3.18951901.2001

Ramette, A., Frapolli, M., Fischer-Le Saux, M., Gruffaz, C., Meyer, J. M., Defago, G., et al. (2011). Pseudomonas protegens sp. nov., widespread plant-protecting bacteria producing the biocontrol compounds 2,4diacetylphloroglucinol and pyoluteorin. Syst. Appl. Microbiol. 34, 180-188. doi: 10.1016/j.syapm.2010.10.005 
Ryder, C., Byrd, M., and Wozniak, D. J. (2007). Role of polysaccharides in Pseudomonas aeruginosa biofilm development. Curr. Opin. Microbiol. 10, 644-648. doi: 10.1016/j.mib.2007.09.010

Sternberg, C., and Tolker-Nielsen, T. (2006). Growing and analyzing biofilms in flow cells. Curr. Protoc. Microbiol. 00:B:1B.2:1B.2.1-1B.2.15. doi: 10.1002/9780471729259.mc01b02s00

Stoodley, P., Sauer, K., Davies, D. G., and Costerton, J. W. (2002). Biofilms as complex differentiated communities. Ann. Rev. Microbiol. 56, 187-209. doi: 10.1146/annurev.micro.56.012302.160705

Waite, R. D., Papakonstantinopoulou, A., Littler, E., and Curtis, M. A. (2005). Transcriptome analysis of Pseudomonas aeruginosa growth: comparison of gene expression in planktonic cultures and developing and mature biofilms. J. Bacteriol. 187, 6571-6576. doi: 10.1128/JB.187.18.6571-6576.2005

Whiteley, M., Bangera, M. G., Bumgarner, R. E., Parsek, M. R., Teitzel, G. M., Lory, S., et al. (2001). Gene expression in Pseudomonas aeruginosa biofilms. Nature 413, 860-864. doi: 10.1038/35101627

Wolfaardt, G., Lawrence, J., Robarts, R., Caldwell, S., and Caldwell, D. (1994). Multicellular organization in a degradative biofilm community. Appl. Environ. Microbiol. 60, 434-446.
Yanisch-Perron, C., Vieira, J., and Messing, J. (1985). Improved M13 phage cloning vectors and host strains: nucleotide sequences of the M13mp18 and pUC19 vectors. Gene 33, 103-119. doi: 10.1016/0378-1119(85) 90120-9

Zhao, K., Tseng, B. S., Beckerman, B., Jin, F., Gibiansky, M. L., Harrison, J. J., et al. (2013). Psl trails guide exploration and microcolony formation in Pseudomonas aeruginosa biofilms. Nature 497, 388-391. doi: 10.1038/nature12155

Conflict of Interest Statement: The authors declare that the research was conducted in the absence of any commercial or financial relationships that could be construed as a potential conflict of interest.

Copyright @ 2015 Periasamy, Nair, Lee, Ong, Goh, Kjelleberg and Rice. This is an open-access article distributed under the terms of the Creative Commons Attribution License (CC BY). The use, distribution or reproduction in other forums is permitted, provided the original author(s) or licensor are credited and that the original publication in this journal is cited, in accordance with accepted academic practice. No use, distribution or reproduction is permitted which does not comply with these terms. 